\title{
Interstitial flow upregulates matrix synthesis and attenuates NF-kB signaling in a novel perfusion bioreactor for articular cartilage tissue engineering
}

\author{
Haneen Abusharkh ${ }^{1}$, Terreill Robertson ${ }^{1}$, Juana Mendenhall ${ }^{2}$, Bulent Gozen ${ }^{1}$, Edwin \\ Tingstad $^{3}$, Nehal Abu-Lael ${ }^{4}$, David Thiessen ${ }^{1}$, and Bernard Van Wie ${ }^{1}$ \\ ${ }^{1}$ Washington State University \\ ${ }^{2}$ Morehouse College \\ ${ }^{3}$ Inland Orthopedic Surgery and Sports Medicine Clinic \\ ${ }^{4}$ The University of Texas at San Antonio
}

October 24, 2021

\begin{abstract}
The present study is focused on designing an easy-to-use novel perfusion system for articular cartilage (AC) tissue engineering and using it to elucidate the mechanism by which interstitial shear upregulates matrix synthesis by articular chondrocytes (AChs). Porous chitosan-agarose (CHAG) scaffolds were synthesized, freeze-dried, and compared to bulk agarose (AG) scaffolds. Both scaffold types were seeded with osteoarthritic human AChs and cultured in a novel perfusion system for one week with a shear-inducing medium flow velocity of $0.33 \mathrm{~mm} / \mathrm{s}$ corresponding to an average surficial shear of $0.4 \mathrm{mPa}$ and a CHAG interstitial shear of $40 \mathrm{mPa}$. While there were no statistical differences in cell viability for perfusion vs. static cultures for either scaffold type, CHAG scaffold cultures exhibited 3.3-fold higher $(\mathrm{p}<0.005)$ cell viability compared to AG scaffold cultures. Effects of combined superficial and interstitial perfusion for CHAG showed 150- and 45-fold ( $<<0.0001)$ increases in total collagen (COL) and 13- and 2.2-fold $(\mathrm{p}<0.001)$ increases in glycosaminoglycans (GAGs) over AG's scaffold non-perfusion and perfusion cultures, respectively, and a 1.5-fold and 3.6-fold $(\mathrm{p}<0.005)$ increase over non-perfusion CHAG cultures. Contrasting CHAG perfusion and static cultures, chondrogenic gene comparisons showed a 3.5-fold increase in collagen type II/type I (COL2A1/COL1A1) mRNA ratio $(\mathrm{p}<0.05)$, and a 1.3-fold increase in aggrecan mRNA. Observed effects are suggested to be the result of inhibiting the inflammatory NF- $x$ B signal transduction pathway as confirmed by a further study that indicated a reduction by 3.2 -fold $(\mathrm{p}<0.05)$ upon exposure to perfusion. Our results demonstrate that the presence of pores plays a critical role in improving cell viability and that interstitial flow caused by medium perfusion through the porous scaffolds enhances the expression of chondrogenic genes and ECM components through the downregulation of NF- $x$ B1.
\end{abstract}

\section{Hosted file}

B\&B_10.18.2021.docx available at https://authorea.com/users/442613/articles/542844interstitial-flow-upregulates-matrix-synthesis-and-attenuates-nf-kb-signaling-in-anovel-perfusion-bioreactor-for-articular-cartilage-tissue-engineering

\section{Hosted file}

Manuscript Figures_10.18.2021.docx available at https://authorea.com/users/442613/articles/ 542844-interstitial-flow-upregulates-matrix-synthesis-and-attenuates-nf-kb-signaling-ina-novel-perfusion-bioreactor-for-articular-cartilage-tissue-engineering 